\title{
Expansion and collapse in the cosmic web
}

\author{
Michael Rauch ${ }^{1}$ \\ ${ }^{1}$ Carnegie Observatories, 813 Santa Barbara Street, Pasadena CA 91101, USA \\ email: mr@ociw.edu
}

\begin{abstract}
In an effort to detect and understand the origin of large scale motions in the gaseous cosmic web at redshifts 2-4.5 we study the kinematics of the intergalactic medium from velocity shifts between absorption systems common to adjacent pairs of lines of sight to background QSO images. We establish the distribution of velocity shear between the lines of sight for different redshifts and transverse spatial separations up to $300 h_{70}^{-1}$ physical kpc. Using a simple analytical model of Lyman $\alpha$ clouds as expanding pancakes, and a cosmological $\Lambda$ CDM hydro-simulation we find that the observed distribution of velocity shear is consistent with an IGM expanding largely with the Hubble flow. The three dimensional distribution of expansion velocities in the hydrosimulation shows that the underlying velocity field is more complex than just simple expansion: the low density gaseous structures responsible for the Lyman $\alpha$ forest are mostly expanding somewhat faster than the Hubble flow, whereas few structures are undergoing gravitational contraction. We also briefly search for traces of galactic feedback and conclude that high redshift superwinds cannot be dominating the movements of the Ly $\alpha$ forest clouds at the observed epoch.
\end{abstract}

\section{Introduction}

How does the cosmic web move? Does it follow the general Hubble flow? Are the motions significantly affected by gravitational collapse, or galactic feedback? In the present contribution we consider the motions of the gaseous cosmic web, as observed in Lyman $\alpha$ forest absorption by the intergalactic medium (IGM). Theoretical ideas imply that Ly $\alpha$ forest spectra are probing mostly the kinematics of flattened gaseous filaments or sheets that follow the general expansion of the Universe. However, strong local departures of the velocity field from isotropy and homogeneity and thus from the Hubble law (e.g. Miralda-Escudé et al. 1996) are suggested by the fact that the clouds selected by Ly $\alpha$ absorption are likely to be already collapsed along one dimension, are drawn from a limited range of (over)densities, and at least sometimes must be under the influence of nearby galactic potential wells, where they may suffer a local gravitational pull or may be exposed to galactic winds. On scales of 10-100 physical kpc, all these effects may lead to observable velocity gradients in the cosmic gas.

We describe here how we can explore these velocity gradients, measuring the shifts between the projected velocities of individual absorption systems common to two adjacent lines of sight (Fig. 1) and relating them to the distribution of intrinsic velocities of the absorbers (Fig. 2, left), as a function of transverse spatial scale and redshift.

\section{The observed distribution of velocity shear}

We used observations of the Ly $\alpha$ forests of two images each of a genuine lensed QSO (RXJ0911.4 $+055 ; \bar{z}=2.57 ; \bar{d}=0.82 h_{70}^{-1}$ proper kpc) and three QSO pairs, including the lower redshift $\mathrm{Q} 2345+007 \mathrm{~A}, \mathrm{~B}\left(\bar{z}=2.04 ; \bar{d}=61 h_{70}^{-1}\right.$ proper $\left.\mathrm{kpc}\right)$ and the two higher redshift pairs Q1422+2309A/Q1424+2255 and SDSSp J143952.58 - 003359.2/SDSSp 

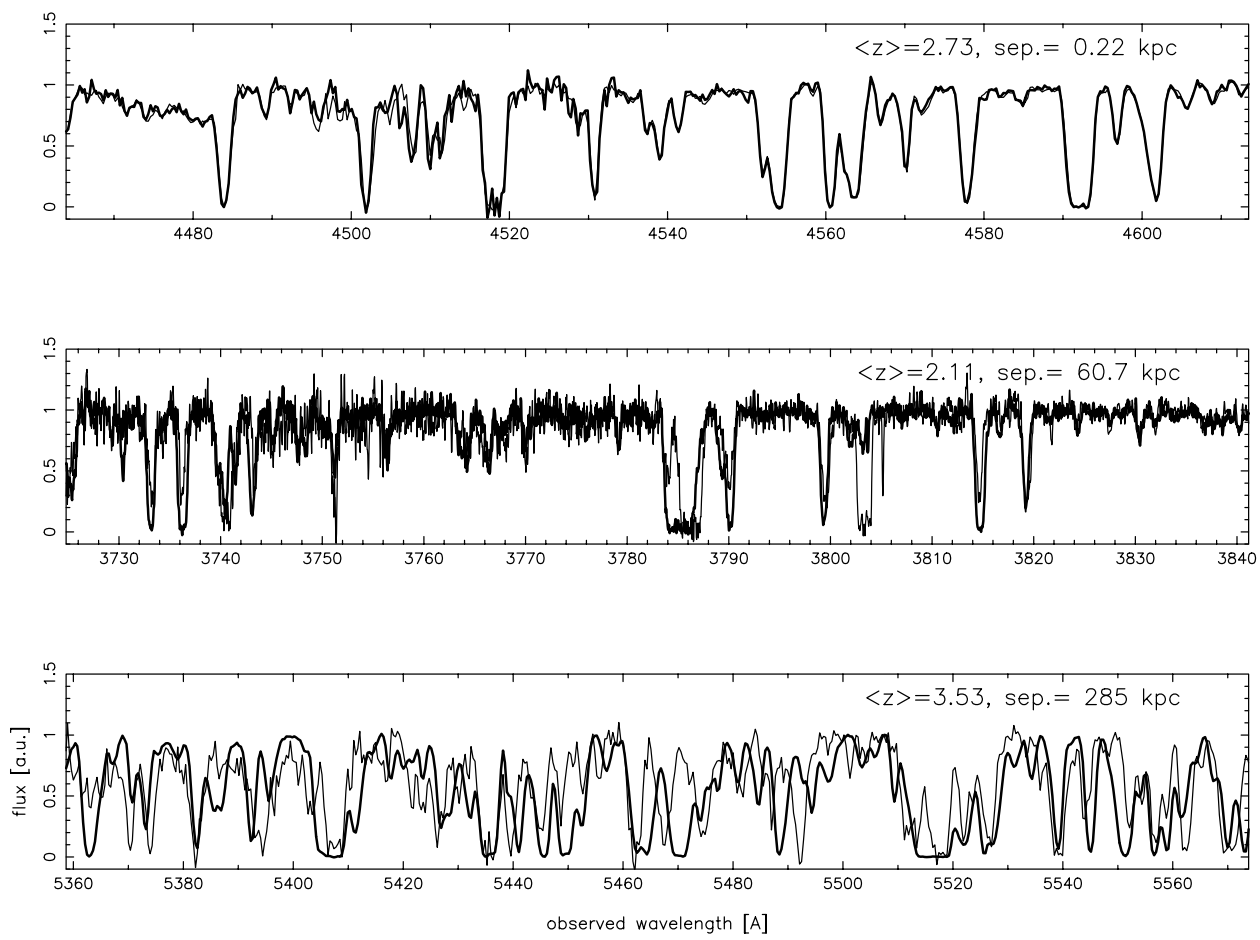

Figure 1. Sections of the three $\operatorname{Ly} \alpha$ forest double lines of sight (each panel consists of the spectra of two lines of sight, over-plotted on top of each other), in the order of increasing separation between the lines of sight. From top to bottom: RXJ0911.4+055, Q2345+007A,B, and the Q1422+2309A,Q1424+2255 pair. The length of the spectra is chosen in all cases to be $100 h^{-1}$ co-moving Mpc. The mean redshifts and the mean beam separation (in physical $h_{70}^{-1}$ $\mathrm{kpc}$ ) are given in the upper-right corner of the spectra. The discrepancies between the column densities and velocities of the individual absorption lines are generally insignificant for the case with sub-kpc beam separation, but they become noticeable at $60 \mathrm{kpc}$ and quite dramatic at 285 kpc. Note that even in the last case there still is quite a bit of similarity between the lines of sight.

J143951.60 - $003429.2\left(\bar{z}=3.62 ; \bar{d}=353 h_{70}^{-1}\right.$ proper kpc, for the combined sample of the high redshift pairs; we shall refer to those two pairs below as the "high redshift" sample). Here $\bar{z}$ and $\bar{d}$ are the mean redshift and proper transverse separation between the lines of sight. Sections of the Ly $\alpha$ forests are shown in Fig. 1.

Shifts between the line-of-sight velocities of individual absorption lines spectra were measured by computing the differences between the flux weighted velocities in both lines of sight. The absorbing regions used were chosen "by eye" in that a suitable wavelength region around each absorption line was delineated with a cursor. This approach may appear anachronistic (why didn't they use an automatic detection algorithm?), but the brain appears superior to the computer in detecting the continuation of an absorption pattern across the lines of sight, amidst regions where a high line density (i.e. at high redshift) and a large separation between the lines of sight produce a large amount of confusion noise. This approach comes at the price of introducing its own staggering selection effects which however, we try to account for by analysing model spectra in precisely the same way as the real data. The distribution of the resulting velocity differences or the "velocity shear" is shown for the three above-mentioned samples on the RHS of Fig. 2. 

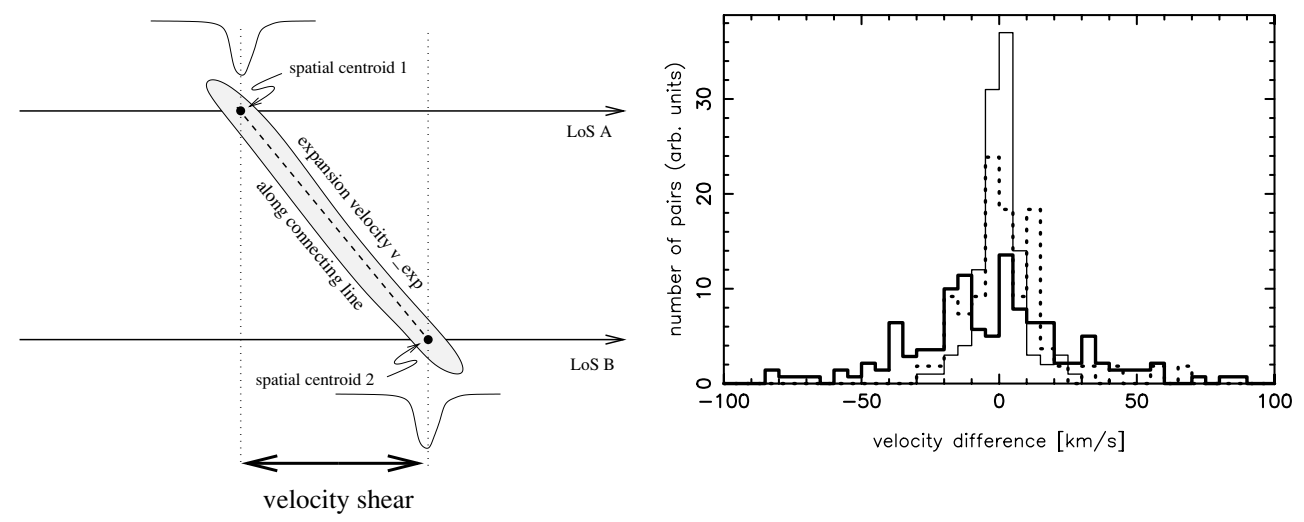

Figure 2. Left panel: the velocity shear across the lines of sight as a projection of the expansion velocity of an absorption-selected gas cloud. (The figure is strictly valid only for pure Hubble flow where the Hubble law guarantees that the angles and positions are the same in position space and velocity space). Right panel: observed distributions of velocity shear normalised to the same integral (arbitrary units) and over-plotted on top of each other. The RXJ0911.4+055 sample is represented by the thin lined histogram, the Q2345+007A,B sample by a dotted one, and the high redshift sample by a thick, solid histogram. At $\bar{z}=2.57 ; \bar{d}=0.82 h_{70}^{-1}$ proper kpc (thin line) the distribution is largely Gaussian and its small width consistent mostly with measurement error, but for the wide separation $\left(\bar{d}=353 h_{70}^{-1}\right.$ proper $\left.\mathrm{kpc}\right)$, high redshift $(\bar{z}=3.62)$ sample the distribution has developed characteristic broad wings not unlike a Lorentzian curve.

\section{Modelling the velocity shear}

What do these distributions of velocity shear mean? Our aim is to relate the velocity shear to the underlying 3-dimensional distribution of expansion velocities of the absorbing clouds, i.e. we want to measure with what fraction of the Hubble flow the Ly $\alpha$ forest structures expand. It is important to realise that this approach is not capable of measuring the absolute value of the Hubble constant $H$, even though we measure absolute values for the velocities. This is because the spatial transverse separation between the lines of sight is computed from the angular diameter distance which is inversely proportional to $H$, whereas the velocity shear is directly proportional to the product of transverse separation and the Hubble constant, so the Hubble constant drops out. Our quantity of interest is the velocity of expansion of a gaseous structure, $v_{e x p}$, relative to the Hubble flow, $v_{\text {Hubble }}$, or the "Hubble ratio" $r=v_{\text {exp }} / v_{H} u b b l e$.

We have modelled the observed distribution in two ways. The first model is a MonteCarlo simulation of randomly oriented, homologously expanding pancakes hit by double lines of sight (see e.g. Haehnelt et al. 1996; Charlton et al. 1995, 1997), which gives a rather good qualitative representation of the shape of the distributions if a (constant) physical radius of $1 / 2 h_{70}^{-1} \mathrm{kpc}$ (D'Odorico et al. 1998) is assumed. The "measured" expansion rates derived from the shear distribution are within $\pm 35 \%$ of the Hubble velocity. The second model is a $\Lambda$ CDM hydro-simulation (Viel, Haehnelt \& Springel 2004). Lines of sight were run through the simulation boxes at redshifts 3.8, 3.4, and 2 using beam separations chosen to match the observations. The simulation does not include galactic feedback. The absorption line regions in the simulation were again chosen "by eye", as for the real data. The resulting shear distribution for the real data, pancake model, and hydro-simulation are shown in Fig. 3.

It can be seen that there is excellent agreement between the simulated and observed distributions of velocity shear, both in terms of shape and width. Kolmogorov-Smirnov 

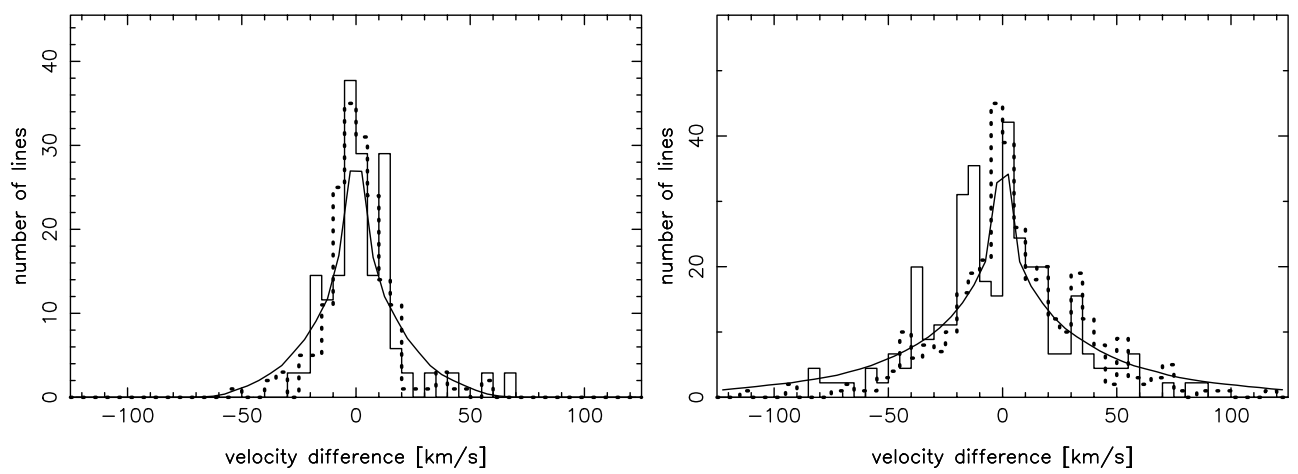

Figure 3. Left: the observed distribution of velocity shear for Q2345+005 (solid histogram), the distribution from the hydro-simulation for $z=2$ (dotted histogram), and the best-fitting expanding pancake model (thick solid line), expanding with 0.8 times the Hubble velocity. Right: same as before but now showing the measurements for the higher redshift $(<z>\approx 3.6)$ combined sample. Again the dotted line is from the simulation for mean redshift $\langle z\rangle=3.6$, and the solid curve is the expanding pancake model for the same redshift, with a Hubble ratio $r=1.0$.

tests show that the distributions are consistent with each other statistically. It appears that whatever produces the distribution in the $\Lambda$ CDM Universe is capable of reproducing the observed velocities.

\section{Motions in the IGM relative to the local Hubble flow}

Working with the simulation as opposed to real data we have additional information available about the underlying physical structures causing a given absorption system and its velocity difference across the lines of sight. In particular, we know the threedimensional peculiar velocities and can identify the spatial positions of the density maxima causing the absorption lines in the adjacent lines of sight. It becomes possible to measure the 3-dimensional expansion velocities along the connecting line between these points (see the scheme in Fig. 2, left panel) and express them in terms of the Hubble ratio. We can ask how those filaments stretch or contract. Fig. 4 gives the distribution of the Hubble ratios for the absorption selected regions (solid histogram) and for randomly chosen directions, i.e. ones included independent of the presence of a Ly $\alpha$ line (dotted histograms). The three panels are for the different redshifts and transverse beam separations. In all three cases, most Ly $\alpha$ forest clouds (based on the median) expand by about $5-20 \%$ faster than Hubble. The mean Hubble ratios are closer to unity, but for the lowest redshift, closest separation case $(z=2.0, \bar{d}=61 \mathrm{kpc})$ (right-most panel) the mean Hubble ratio has dropped to 0.8. Apparently, the distribution has become increasingly dominated by contracting clouds. The sequence from left to right is not a straightforward evolutionary sequence because of the different transverse separations. In addition, there is a redshift dependent density threshold, because the constant column density detection threshold imposed selects higher density regions at lower redshift.

To demonstrate that the entities selected by their Ly $\alpha$ forest absorption are not just random regions of the Universe, the distribution of Hubble ratios was computed also for regions irrespective of whether they produced absorption systems or not. Those distributions are plotted as the dotted histograms in Fig. 4. They are similar to the absorptionselected regions for the highest redshift bin (left-most panel in Fig. 4), but are significantly shifted already in the middle panel $(z=3.4)$. The right-most panel shows that random 

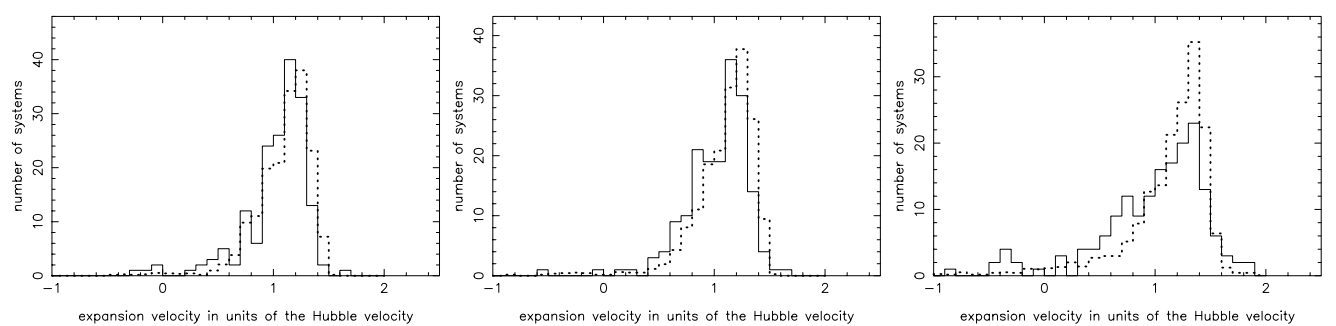

Figure 4. Top: expansion velocity in the $\Lambda$-CDM simulation, in units of the Hubble velocity, measured along the straight line connecting the spatial absorption centroids (Fig. 2, left panel). The histograms belong to (from left to right) the $z=3.8, z=3.4$, and $z=2.0$ simulations (solid lines). Note the "super-Hubble" peak and the "sub-Hubble" tail indicating break-away from the general expansion. The dotted histograms apply to the expansion velocity measured at random positions along the line of sight (i.e. irrespective of there being an absorption line).

regions do not partake in gravitational collapse as strongly as the absorption selected ones, but that they continue to expand with super-Hubble velocities. Random regions are of course bound to mainly lie in cosmic voids and are expected to expand faster than Hubble, so this behaviour is no surprise. The fact that most Ly $\alpha$ forest clouds also expand with super-Hubble velocities is perhaps less obvious but it is consistent with them being filaments that are pulled by and draining into the more massive nodes connecting the cosmic web. Another, equivalent explanation recognises that Ly $\alpha$ clouds, being the boundaries of voids, have to expand faster than Hubble, too.

\section{Galactic superwinds?}

It appears that the standard Hubble expansion, modified by gravitational instability can account satisfactorily for the observed velocities. It is tempting to investigate whether there is still room for significant galactic feedback to have contributed to the kinematics of the cosmic web. Occasionally, rather far-ranging claims have been promoted on the conference circuit about the impact of galactic superwinds on the properties of the intergalactic medium. Extrapolations of the properties of Lyman Break galaxy winds have led to suggestions that cosmological conclusions based on Ly $\alpha$ forest observations could be in error if interpreted solely from within the gravitational instability picture. Fortunately, Adelberger (these proceedings) reminds us that there is really no factual basis for such assumptions, as the volume filling factor of Lyman break superwinds with respect to the Universe is quite small. In other words (my words) Lyman break superwinds are just storms in intergalactic teacups. Nevertheless, the idea that winds of a more general nature (perhaps from dwarf galaxies, at higher redshift) could have played a significant role is suggested by the widespread metal enrichment in the IGM at the highest redshifts we can currently observe (e.g. Songaila 2001) and by the observed mass-metallicity relation of galaxies (Tremonti et al. 2004). If the IGM has been widely polluted by winds it is conceivable that they also left a kinematic imprint on it.

To test the idea that the Ly $\alpha$ forest is globally disturbed by winds we performed Monte-Carlo simulations of expanding wind bubbles, the shells of which were considered to give rise to absorption in double lines of sight (Fig. 5, left panel). The differences in projected velocity between the lines of sight caused by the curvature of the expanding shell were then compared with the observed distribution of velocity shear.

The small widths of the observed distribution (about $17 \mathrm{kms}^{-1}$ at $z \sim 2$ over $60 \mathrm{kpc}$ ) only admits winds (Fig. 5 right panel) with expansion velocities less than between 45 

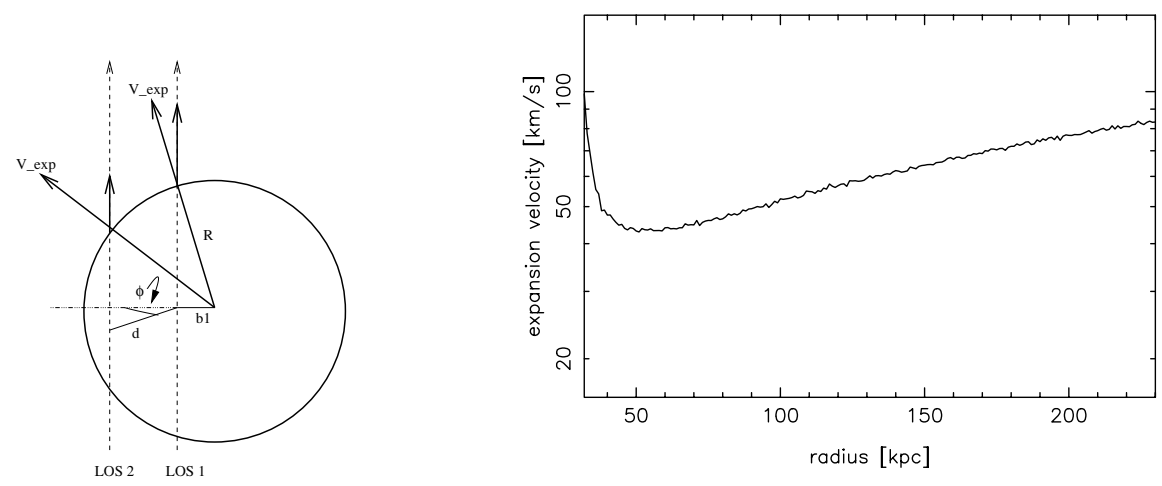

Figure 5. Left panel: velocity shear arising from projection effects when an expanding bubble is intersected by two lines of sight to background QSOs. Right panel: maximum expansion velocity as a function of radius for expanding $z=2$ bubbles capable of producing the mean of the observed $z=2$ distribution of velocity differences.

and $85 \mathrm{kms}^{-1}$, and even that only if the observed velocity shear is entirely ascribed to winds, which is unlikely to be true. However, our measurement only constrains a global population of winds and would show only a detectable contribution if winds occupied on the order of $10-20 \%$ or more of the volume of the structures making up the Ly $\alpha$ forest. Thus one possible conclusion is that the filling factor of detectable galactic winds (Lyman break or not) for the over-dense regions making up the Lyo forest is small at the time of the observations. The cosmic volume filling factor could still be larger if winds vented preferentially into voids, but it could also be smaller if the wind producing galaxies are clustered. The other possible conclusion is that winds do indeed have a large filling factor but have faded or expired by the time we observe the forest. An early widespread population $(z>5)$ of winds responsible for much of the metal enrichment of the IGM by volume would not be ruled out by our measurement; there appears to be sufficient time for the products of $z \sim 6$ winds to have slipped back sub-sonically into their original dark matter potential wells by the time we observe the Ly $\alpha$ forest.

\section{Acknowledgements}

I acknowledge the work of my collaborators George Becker, Matteo Viel, Wal Sargent, Alain Smette, Rob Simcoe, Tom Barlow, and Martin Haehnelt. The research sketched here and further discussion and references will be presented in a forthcoming paper.

\section{References}

Charlton, J. C., Anninos, P., Zhang, Y., Norman, M. L., 1997, ApJ, 485, 26

Charlton, J. C., Churchill, C. W., Linder, S. M., 1995, ApJ, 452, L81

D’Odorico, V., Cristiani, S., D’Odorico, S., Fontana, A., Giallongo, E., Shaver, P., 1998, A\&A, 339,678

Haehnelt, M. G., 1996, in: M. Bremer et al. (eds.), Cold Gas at High Redshift, (Dordrecht: Kluwer)

Miralda-Escudé, J., Cen, R., Ostriker, J. P., Rauch, M., 1996, ApJ, 471, 582

Songaila, A., 2001, ApJ, 561, 153

Tremonti, C. A., et al., 2004, ApJ, 613, 898

Viel, M., Haehnelt, M. G., Springel, V., 2004, MNRAS, 354, 684 